\title{
Use of retroviral and lentiviral vectors to deliver new gene therapies
}

Volume 5 Issue I - 2017

\author{
Keywords: Lentivirus, Retrovirus, shRNA, CRISPR/Cas9, Gene \\ editing
}

Abbreviations: MLV, Murine Leukemia Virus; shRNA, Short Hairpin RNA; CRISPR-Cas9, Clustered Regularly Interspaced Short Palindromic Repeat/CRISPR-Associated Protein (Cas)9

\section{Introduction}

Human genetic variations are commonly the cause of numerous malignancies and diseases. Genome-wide association studies uncovered new correlations between genetic mutations and disease incidence. ${ }^{1}$ A plethora of whole genome association studies permitted the examination of numerous common genetic variants in different individuals to assess its association with a genetic trait and the pathological consequences. Researchers recognized that the observed genetic heterogeneity amongst individuals and genetic traits constitutes a Herculean challenge to develop new treatments. With this new personalized medicine information approach, the advance in genetic engineering was fast to act to try and help solving genetic abnormalities. Understanding the observed functional genetic diversity helps to correlate pathology phenotypes with the specific responses to therapeutic treatments. Finding the causal genetic mutations is crucial to define a precise strategy to correct the genetic imperfection. Retroviruses and lentiviruses were soon considered a viable approach to manipulate the human genome taking advantage of the mechanisms viruses use to invade and exploit the host genome.

The use of retroviral vectors is one of the most common methods to develop novel gene therapy approaches. Recombinant retroviruses such as the murine leukemia virus (MLV) have the capacity to integrate into the host genome in a stable manner. ${ }^{2}$ The stable integration of the engineered vector provides a potential long term therapeutic gene expression to treat patients. Due to its powerful efficiency to alter the genome, it is necessary to be sure that retroviral vectors integration sites are not random in the mammalian genome. A genome wide analysis of retroviral DNA integration in tumors genome identified hotspots of MLV integration. Studies of MLV integration sites in culture cells lines suggest that integration sites preferentially occur near accessible chromatin regions. ${ }^{3}$ Analysis of the MLV integration between tumors indicated that the convergence of integration sites is not a rare event, and that the integration mutagenesis process might be unique between tumor mutagenesis mechanisms. ${ }^{4}$

Regardless of its flexible application in gene therapy, the use of retroviruses is limited to the infection of mitotically active cell types whereas lentiviruses are able to infect non-dividing and actively dividing cells. Lentiviral vectors based on the human immunodeficiency virus type- 1 take advantage of the accessory proteins to enable the integration in the absence of cell division. ${ }^{5}$ Lentivectors do not necessarily require active cell division for proviral integration and that is a significant advantage $e^{6,7}$ because gene delivery can be achieved without the requirement of cell growth factors

\author{
Alexandre Goncalves \\ University Zurich, Switzerland \\ Correspondence: Alexandre Goncalves, University Zurich \\ Leuengasse 25, Basel, Switzerland, Tel 00417640399 35, \\ Email alexander.goncalves@gmx.ch \\ Received: January 27, 2017 | Published: January 31, 2017
}

administration to induce cell proliferation in cells transduced ex vivo. Furthermore, engineered lentivectors can also be administered in vivo to facilitate the transduction of cells that are usually unresponsive to other types of gene therapy vectors. Recombinant lentiviral vectors are powerful gene transfer tools that can be used in the central nervous system $^{8}$ and have the potential to deliver long-term new treatments for neurological disorders, such as the Parkinson's disease. ${ }^{9}$

The use of lentiviral gene delivery vectors in cancer research studies takes advantage of the fact that proviral DNA integrates into the genome of infected cells. That confers the crucial heritability property to the newly introduced genetic alterations. Researchers can use lentiviruses to deliver short hairpin RNA (shRNAs) to silence key oncogenes and to assess cancer development. Scientists administered injections of lentiviruses co-expressing shRNAs against Trp53 and Nf1 to successfully generate mouse models of glioma. ${ }^{10}$ Loss-of-function experiments using pooled shRNA libraries can target multiple genes during tumorigenesis to evaluate their potencial value as therapeutic targets in vivo to develop new therapies. ${ }^{11-13}$ The shRNA screens can identify relevant tumor suppressor genes and biological pathways to establish new tumor models. ${ }^{14}$

With the identification of new genetic fragilities and potential correction regions it was mandatory to establish new strategies to deliver the necessary genetic corrections. A new RNA-guided endonuclease based genome editing method called clustered regularly interspaced short palindromic repeat (CRISPR)/CRISPR-associated protein (Cas) 9 system was rapidly developed. ${ }^{15}$ The CRISPR-Cas 9 method is based on the bacterial mechanism that cleaves foreign nucleic acids as an immune response. ${ }^{16}$ The CRISPR RNA and a trans-activating RNA guide the Cas9 endonuclease to a specific DNA target sequence to generate double-strand break (CRISPR/Cas-based methods for genome engineering. Therefore, lentiviruses pre-packed with the CRISPR/Cas9 system offer a significant opportunity for human gene therapy. Cas9-mediated therapeutic approaches include targeted genome editing to correct genetic disorders and targeted genome regulation to modify endogenous protein levels. However, CRISPR/Cas9 gene therapy abilities cannot overlook the occasional 
off-target effects. Those nucleases can induce off-target mutations and undesirable chromosomal translocations associated with off-target DNA cleavages. ${ }^{17}$

A stable and irreversible gene therapy method such as Crispr/ Cas9 delivered by lentiviruses is an important medical advance. It has the potencial to cure otherwise irreversible diseases but unexpected off-target effects risks the life of patients. Scientists investigated the nature of bacterial CRISPR-Cas systems in detail and discovered a new method to regulate the system. The discovery of Crispr/ Cas9 inhibitor proteins that are able to block the commonly used Streptococcus pyogenes Cas9 can be used to regulate the genome engineering activities of CRISPR/Cas9. Those proteins can act as post-translational "off-switches" for Cas9 and control the Crispr/ Cas9 genome engineering system. The same way CRISPR/Cas9 must be regulated, lentiviral vectors must also be controlled to prevent unwanted effects. It is important to remember that safety and ethics for HIV-based lentivectors must be highly regulated. Using this gene therapy method in HIV-negative patients may result in a partial seroconversion.

\section{Acknowledgements}

The author expresses his appreciation to Prof. Ian Frew and the Epithelial Cancers research group at the Institute of Physiology of the University of Zurich.

\section{Conflicts of interest}

None.

\section{References}

1. Stadler ZK, Thom P, Robson ME, et al. Genome-Wide Association Studies of Cancer. J Clin Oncol. 2010;28(27):4255-4267.

2. Hu WS and Pathak VK. Design of retroviral vectors and helper cells for gene therapy. Pharmacol Rev. 2000;52(4):493-511.

3. Bushman F, Lewinski M, Ciuffi A, et al. Genome Wide Analysis of retroviral DNA integration. Nat Rev Microbiol. 2005;3(11):848-858.

4. Tsuruyama T, Hiratsuka T, Yamada N. Hotspots of MLV integration in the hematopoietic tumor genome. Oncogene. 2016.
5. Cooray S, Howe SJ, Thrasher AJ. Retrovirus and lentivirus vector design and methods of cell conditioning. Methods Enzymol. 2012;507:29-57.

6. Naldini L, Blömer U, Gallay $\mathrm{P}$, et al. In vivo delivery and stable transduction of nondividing cells by a lentiviral vector. Science. 1996;272(5259):263-267.

7. Miller DG, Adam MA, Miller AD. Gene transfer by retrovirus vectors occurs only in cells that are actively replicating at the time of infection. Mol Cell Biol. 1990;10(8):4239-4242.

8. Kay MA, Glorioso JC, Naldini L. Viral vectors for gene therapy: the art of turning infectious agents into vehicles of therapeutics. Nat Med. 2001;7(1):33-40.

9. Kordower JH, Emborg ME, Bloch J, et al. Parkinson's disease: neurodegeneration prevented by lentiviral vector delivery of GDNF in primate models of Parkinson's disease. Science. 2000;290(5492):767-773

10. Friedmann-Morvinski D, Bushong EA, Ke E, et al. Dedifferentiation of neurons and astrocytes by oncogenes can induce gliomas in mice. Science. 2012;338(6110):1080-1084.

11. Gargiulo G, Serresi M, Cesaroni M, et al. In vivo shRNA screens in solid tumors. Nat Protoc. 2014;9(12):2880-2902.

12. Guinot A, Lehmann H, Wild PJ, et al. Combined deletion of Vhl, Trp53 and Kif3a causes cystic and neoplastic renal lesions. J Pathol. 2016;239(3):365-373.

13. Frew IJ. Tumour modelling using viral vectors. Oncotarget. 2015;6(16):13854-13855.

14. Deans RM, Morgens DW, Ökesli A, et al. Parallel shRNA and CRISPRCas9 screens enable antiviral drug target identification. Nat Chem Biol. 2016;12(5):361-366.

15. Jinek M, Chylinski K, Fonfara I, et al. A programmable dual-RNAguided DNA endonuclease in adaptive bacterial immunity. Science. 2012;337(6096):8160-821.

16. Wiedenheft B, Sternberg SH, Doudna JA. RNA-guided genetic silencing systems in bacteria and archaea. Nature. 2012;482(7385):331-338.

17. Cho SW, Kim S, Kim Y, et al. Analysis of off-target effects of CRISPR/ Cas-derived RNA-guided endonucleases and nickases. Genome Res. 2014;24(1):132-141. 